\title{
PRE-WHITE DWARF EVOLUTION THROUGH PLANETARY NEBULAE
}

\author{
FRANCESCA D'ANTONA \\ Osservatorio Astronomico di Roma \\ I-00040 Monte Porzio \\ Italy \\ ITALO MAZZITELLI \\ Istituto di Astrofisica Spaziale CNR \\ C.P. 67 \\ I-00044 Frascati \\ Italy
}

\begin{abstract}
In the present review we summarize the problems relative to the chemical composition of the inner and outer layers of white dwarfs as expected from stellar evolution. We point out that there is a contrast between standard predictions and the indications deriving from studies of white dwarfs, as "massive" hydrogen remnat layers seem not to be present on single white dwarfs. We discuss a previously neglected feature of stellar models in the phase of thermal pulses -which occurs when the outer hydrogen envelope becomes very small- by which the progenitors of low total mass may get rid of practically the whole hydrogen envelope during the final phases of asymptotic giant branch evolution. We finally propose a new global scheme for the pre-white dwarf evolution, which depends mainly on the initial progenitor mass.
\end{abstract}

\section{Introduction}

The present review deals with the following question: which is the chemical composition (stratification) expected for single White Dwarfs (WDs) on the basis of today's knowledge of stellar evolution? It is noteworthy to remark that, while the attention of this meeting is focused on the surface stellar composition of stars as a signature of the evolution which has been responsible for the observed abundances, study of WDs has shown that the chemistry of these stars, in the surface layers even more than in the inner ones, determines their stellar evolution properties, namely their cooling times down to invisibility (Mazzitelli and D'Antona 1987, Winget and Van Horn 1987, D'Antona and Mazzitelli 1989, 1990). There are no other stellar structures for which the composition is so intimately linked to the evolution! What WD researchers would like to know from stellar evolution can be articulated, in first approximation, into the following questions:

1. what is the carbon to oxygen $(\mathrm{C} / \mathrm{O})$ ratio expected for the inner regions of WDs?

2. how massive $\left(\mathrm{M}_{\mathrm{He}}\right)$ is the helium remnant layer expected to be?

3. what range of masses $\left(M_{H}\right)$ do we expect for the hydrogen layer of DA type WDs?

Let us also add these other questions to show the problem in its more general form: 
1. In which way the $\mathrm{C} / \mathrm{O}$ ratio and $\mathrm{M}_{\mathrm{He}}$ are expected to depend on the mass $\left(\mathrm{M}_{\text {prog }}\right)$ and initial chemical composition of the progenitor?

2. Do we have any good reasons to expect a dependence of the emerging WD spectral type on $\mathrm{M}_{\text {prog }}$ ?

In the latest years many researchers have devoted efforts to post-Asymptotic Giant Branch (AGB) evolution, also with the aim to clarify these issues, but some of the answers are still clearly unsatisfactory. Personally we have devoted three other reviews at least partially to this subject (Mazzitelli and D'Antona 1987, Mazzitelli 1989, D'Antona 1989), and this last review complements the others.

In the following we first summarize briefly the problem of the $\mathrm{C} / \mathrm{O}$ ratio in the interior of WDs. After, we review the present standard scheme of post-AGB evolution (Schönberner 1979, 1981, 1983, 1987, Iben 1984, Iben and Mc Donald 1985 and 1986 -for an update comprehensive review see Iben 1989). This scheme predicts that most of DAs (WDs having hydrogen atmosphere) have evolutionary $\mathrm{M}_{\mathbf{H}}\left(\sim 10^{-4} \mathrm{M}_{\odot}\right.$ for a typical $0.6 \mathrm{M}_{\odot}$ WD). This is in contradiction with several different indications coming from study of WDs, by which $\mathrm{M}_{\mathrm{H}}$ on most DAs is concluded to be thin $\left(\lesssim 10^{-8} \mathrm{M}_{\odot}\right)$ or even very thin $\left(\lesssim 10^{-14} \mathrm{M}_{\odot}\right)$ (see D'Antona 1987 and Fontaine and Wesemael 1987, 1990) for summaries of these problems.

Based on consideration of recent stellar models results (Mazzitelli 1990) we suggest a possible escape form the standard scheme, which allows formation of WDs with very low hydrogen remant layers and predicts observational consequences regarding the status of H-rich Planetary Nebulae Nuclei (PNN).

We review the present theories for the evolution to WDs of relatively massive progenitors $\left(\mathrm{M} \gtrsim 4-5 \mathrm{M}_{\odot}\right)$, consistently with the observations of lack of very luminous carbon stars and with the observations of $M$-supergiants in the Magellanic Clouds.

We finally summarize what we consider the present status of the relation between $M_{\text {prog }}$ and emerging spectral type, on the basis of the proposed suggestions.

\section{The interior chemistry of WDs}

Before we touch the subject of the $\mathrm{C} / \mathrm{O}$ ratio in common WDs, two warnings must be kept in mind:

i) Bergeron et al. (1990) have revised the mass distribution of DA WDs. While Weidemann and Koester (1984) determined as average mass $\left\langle M>\sim 0.58 \mathrm{M}_{\odot}\right.$ by multichannel photometry, the new results suggest $\left\langle M>\sim 0.53 \mathrm{M}_{\odot}\right.$. Taken at face value, the recent result would imply that mass loss during the Giant Branch and AGB evolution of low mass stars is more efficient than previously considered. Thus, a significant fraction of single WDs - possibly those belonging to the most metal rich stellar populations, for which mass loss is mostly important-could be remnants of progenitors which were not able to ignite the helium core flash. In this case, their chemical stratification is very simple: $\mathrm{M}_{\mathrm{He}} \sim \mathrm{M}_{\mathrm{WD}} \sim 0.4-0.45 \mathrm{M}_{\odot}$, and $\mathrm{M}_{\mathrm{H}} \sim 10^{-3} \mathrm{M}_{\odot}$.

ii) For progenitors close to the minimum mass for supernova explosion $\left(M_{u p}\right)$ carbon ignition may be able to remove core degeneracy, and form a Oxygen-Neon-Magnesium core. Mass loss before collapse may leave a $\mathrm{O}-\mathrm{Ne}-\mathrm{Mg}$ WD of $\mathrm{M} \sim 1.2-1.37 \mathrm{M}_{\odot}$ (Nomoto 1984). At least in nova systems we know that such WDs do exist (Williams 1985 , Truran and Livio 1989), although we can not be sure that there are any single WDs with such a core.

Common WDs are Carbon-Oxygen, and the core contains more than $95 \%$ of the mass. The core composition is fundamental for the further evolution in the WD stage. In fact, the cooling time depends on the thermal energy content of the WD, inversely proportional to the average atomic weight of the constituents. The chrystallization temperature also depends on $Z^{2} A^{-1 / 3}$, and the possible occurrence of separation between Oxygen and Carbon at crystallization (Stevenson 1980, Garcia Berro et al. 1988) which may prolongate the 
cooling times by billions of years, need an exact knowledge of the $\mathrm{C} / \mathrm{O}$ stratification. This stratification is the result of three main phases:

1. core helium burning

2. thick helium shell burning

3. thermal pulses (TPs)

Furthermore, apart from semiconvection, which in any case needs to be considered, conditions at the border of the convective core favour the onset of "extra-mixing" processes -such as breathing pulses or overshooting- which inject fresh ${ }^{4} \mathrm{He}$ into the core towards the end of core-helium burning, and this also is reflected into the chemical stratification. The result of helium burning depends on the ratio of the reaction rates $3 \alpha$ to ${ }^{12} \mathrm{C}+\alpha$, and thus not only on the rate of the cross sections but also on the ratio $\left(\mathrm{X}_{4} \mathrm{He}\right)^{2} \rho / \mathrm{X}_{12} \mathrm{C}$. The reaction ${ }^{12} \mathrm{C}+\alpha$ dominates when the helium content $\mathrm{X}_{4_{\mathrm{He}}}$ is low. This is the reason why the late phases of burning and the possible extra-mixing, which injects fresh helium into the carbon rich core is crucial for the resulting $\mathrm{C} / \mathrm{O}$ ratio. The recent computations relative to the ${ }^{16} \mathrm{O}$ abundance in the He-exhausted core of a $1 \mathrm{M}_{\odot}$ progenitor are summarized in the following table.

$\begin{array}{cccc}\text { Reference } & \sigma\left({ }^{12} \mathrm{C}+\alpha\right) & { }^{16} O & { }^{16} O \text { after extra-mixing } \\ \text { Fowler } \text { et al. } 1975 & \mathrm{~F} & 50 \% & 60-70 \% \\ \text { Harris } \text { et al. } 1983 & \mathrm{~F}^{*} 3 & 70 \% & \gtrsim 80 \% \\ \text { Caughlan and Fowler } 1988 & \sim \mathrm{F}^{*} 2 & 60 \% & \sim 75 \%\end{array}$

We have listed only reference to the expressions for the cross section from literature compilations. For a recent discussion of the uncertainties in the experimental results see Barnes (1988). The cross section ${ }^{12} \mathrm{C}+\alpha$ has fluctuated in recent years from the original value reported in Fowler et al. 1975 to values up to four times larger. The present (but not latest) expression adopted by Caughlan and Fowler (1988) is corresponding to about a factor two larger than the original one. The table shows that the influence of extra-mixing at the core boundary in the final phases is at least as important as a factor two difference in the cross section determination.

In view of the behaviour of the ratio between the cross-sections as a function of the temperature (see, e.g., Mazzitelli and D'Antona 1987), the golden rule to understand the results of burning is the following: the lower the burning temperature, the larger is the resulting oxygen content. Thus the regions emerging from shell burning, which takes place at larger temperature, have $\mathrm{C} / \mathrm{O}$ larger than the core, and $\mathrm{C} / \mathrm{O}$ is smaller for smaller $\mathrm{M}_{\text {prog }}$, which have lower burning temperature. The dependence of the stratification on $\mathbf{M}_{\text {prog }}$ given in Mazzitelli and D'Antona $1986 \mathrm{~b}$ can be considered reasonably updated, as those results were obtained by adopting the Fowler et al. cross section multiplied by a factor 2 .

A powerful check of the core composition of WDs is in principle available, through the study of the period derivatives of pulsating DA WDs (ZZ Ceti variables). In this class of pulsators, the period should be linked to the thermal timescale $\left(t_{t h}\right)$ at the base of their convective envelope (Cox 1980). While the star cools, the bottom of convection enters deeper into the envelope, $t_{t h}$ increases and so the period of pulsation is expected to increase. Determination of period derivatives is a long-lasting job, as observations require a long time baseline, but already one $\dot{P}$ is available through the operation of the "Whole Earth Telescope" (Nather et al. 1990) for the star G117-B15A (Kepler et al. 1990) and the results are under comparison with models. 


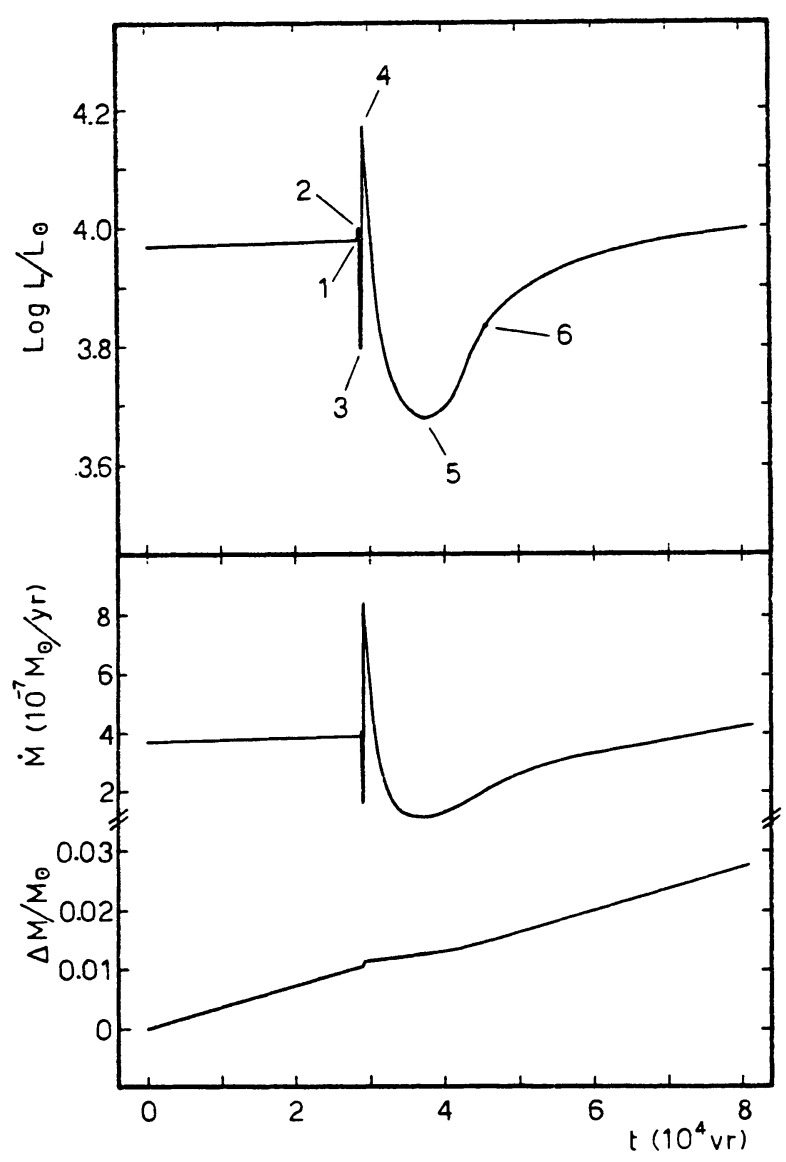

Figure 1: Run of luminosity, mass loss rate and cumulative mass lost during a typical thermal pulse, starting and ending at the point in which helium burning is at minimum. Several stages are indicated: 1) onset of He-burning in a convective shell; 2) Maximum $\mathrm{L}_{\mathrm{He}}$; 3) $\mathrm{L}_{\mathrm{He}}=\mathrm{L}$; 4) Maximum extension of external convection; 5) Hydrogen burning already resumed; 6) $\mathrm{L}_{\mathrm{He}}=10 \% \mathrm{~L}$.

\section{The helium remnant envelope}

In principle, the helium mantle of Carbon-oxygen WDs, $\mathrm{M}_{\mathrm{He}}$, must bear connection with the helium intershell mass $\left(M_{i . s .}\right)$ which the progenitor had at the end of the TP phase. For this discussion and for the following, let us examine the detailed behaviour of stars during TPs. Top of figure 1 shows the luminosity evolution of an AGB star of $3 \mathrm{M}_{\odot}$ during a typical TP (from Mazzitelli 1987). The total H-mass processed into Helium between two consecutive pulses will be referred to as $\Delta \mathrm{M}_{\mathrm{h}}$. At (1) the helium pulse begins with the onset of a convective helium shell, and already at (2) $\mathrm{L}_{\mathrm{He}}$ is at its maximum: as we see, nothing has happened at the surface, but expansion and consequent rapid switch off of the H-burning shell produces a minimum in the stellar luminosity, which then raises by a factor $\sim 2$, while the hydrogen external convection reaches its maximum extension (4). Afterwards, there 


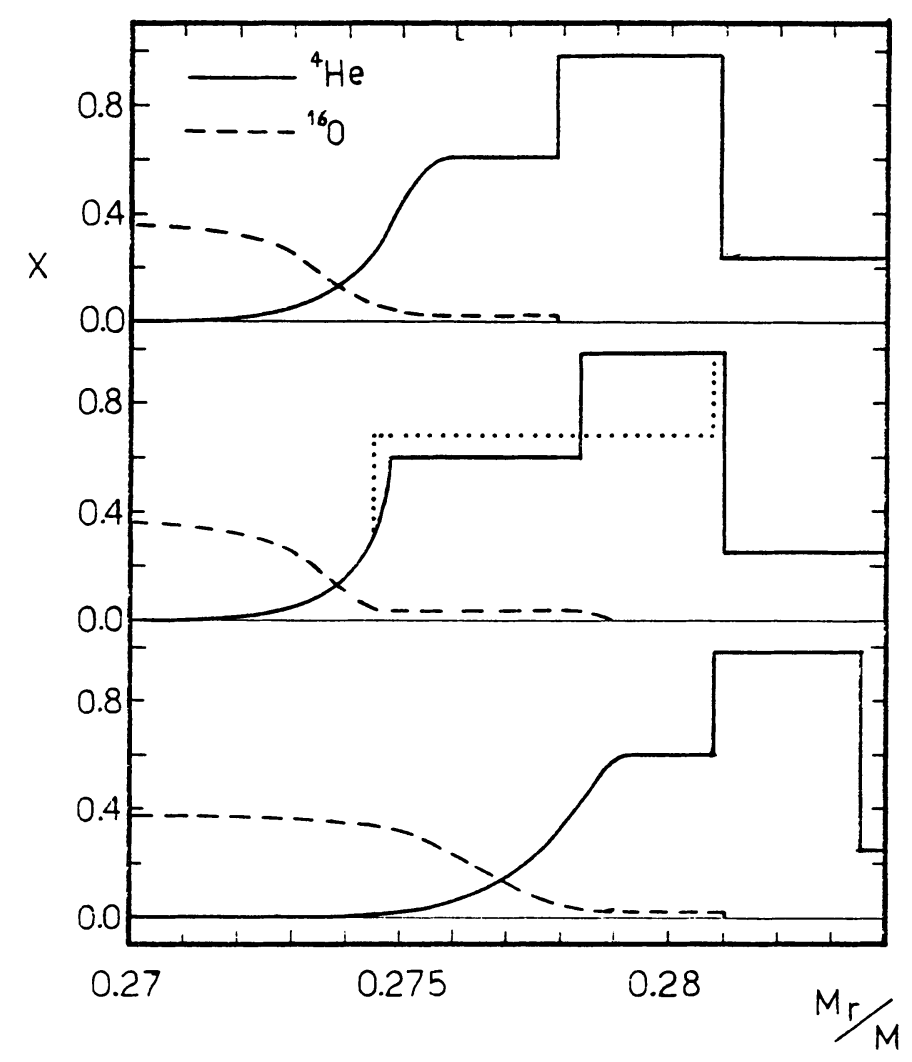

Figure 2: helium and oxygen profile at several stages of a typical TP. The upper part is just before a TP; the middle part shows the onset of convective He-burning (continuous line) and its maximum extension (dotted), which also corresponds to the minumum $\mathbf{M}_{\mathrm{buff}}$; the lower part shows the effect of quiescent He- and H-burning in the phase just preceding the next pulse. The computations refer to a core mass of $0.7 \mathrm{M}_{\odot}$. No dredge up is present.

is a prolonged phase of quiescent He burning, and at point 5 hydrogen burning is active again, and slowly becomes more important then He-burning, which provides only $\sim 10 \% \mathrm{~L}$ at point 6 . From this summary description, we can derive the following conclusion: if the WD stratification is "frozen" out of the last $T P$, the maximum $M_{i . s}$. will be left when the evolution is stopped just before the next TP, at point (1), the minimum one if it is stopped following the phase of stationary helium burning, around point (5). For a typical core of $0.6 \mathrm{M}_{\odot}$ thus $0.015 \lesssim \mathrm{M}_{\mathrm{i} . \mathrm{s} .} \lesssim 0.02 \mathrm{M}_{\odot}$. Dredge up may further reduce these values by $\sim 30 \%$.

As shown in figure 2, where the helium abundance profile at different stages of TPs is presented, $\mathbf{M}_{\mathrm{i} . \mathrm{s} .}$ is made up of two parts: an almost pure helium buffer $\left(\mathrm{M}_{\text {buff }}\right)$ which results from hydrogen shell burning, and a carbon rich layer $\left(\mathrm{M}_{\mathrm{He}-\mathrm{C}}\right)$ whose maximum outward extension is achieved at the beginning of each TP, when the onset of convection spreads into the convective shell the carbon accumulated at the border of the core. In the WD stage, the helium mantle will be: $\mathrm{M}_{\mathrm{He}}=\mathrm{M}_{\mathrm{buff}}+\mathrm{M}_{\mathrm{He}-\mathrm{C}}-\int_{0}^{M_{\mathrm{He}-\mathrm{C}}} \mathrm{X}_{12 \mathrm{C}} \times \mathrm{dM}$, as gravitational settling will separate carbon from helium. So $\mathbf{M}_{\mathrm{He}}$ on the WD is $\sim 30 \%$ smaller than $\mathbf{M}_{\mathrm{i} . \mathrm{s} .}$. 
The minimum $\mathrm{M}_{\mathrm{He}}$ on a typical WD is thus expected to be $\sim 2 \times 10^{-3}$. Whether this is in contrast with the current explanation of DQ type WDs (Pelletier et al. 1986) is discussed in D'Antona 1989.

It is important to notice that the recent analysis of four members of the PG1159 class of very hot WDs shows that their atmospheres are dominated by helium, carbon and oxygen (Werner et. al. 1990). The oxygen abundance profile during the TP is also shown in figure 2. The flash starts at the base of the helium layer, where a large fraction of carbon and some oxygen are already present, so that part of the carbon of the convective shell is produced during the pulse itself, but part is simply convected outwards from inner regions. Although practically no oxygen is produced by helium burning during the TP in the convective shell, oxygen had been produced during the late phases of the preceding pulse, during stationary helium burning which exhaustes helium. Thus a small quantity of oxygen $(\lesssim 4 \%)$ can be found also into $\mathrm{M}_{\mathrm{He}-\mathrm{C}}$. Towards the final stage of an interpulse period, this $\mathrm{M}_{\mathrm{He}-\mathrm{C}}$ will have obtained a carbon abundance profile which reflects the prolonged quiescent phase of He-shell burning following the peak of the pulse. The presence of oxygen at very large levels ( $\sim 17 \%$ in mass fraction) in the PG type WDs implies that these stars have been able to expose the even more internal parts of the helium intershell, close to the region in which the latest TP must have occurred (Mazzitelli and D'Antona 1990). Both careful consideration of model atmospheres and of evolution is here necessary, as the inner intershell is already at WD dimensions, and has therefore a large binding energy even during the TP.

\section{The hydrogen remnant}

The standard description of post-AGB evolution and of the different emerging spectral types provides a link between the phase during the TP at which departure from the AGB occurs and the final detailed evolution to WD. In this scheme, the main controlling quantity is the mass contained in the pure-helium buffer $\left(M_{\text {buff }}\right)$, which determines whether hydrogen will be left or not on top of the WD. This description has emerged in the course of the latest 10 years, due to the efforts of several researchers, including Schönberner, Iben, Wood and Faulkner, and ourselves, and is summarized with care in Iben (1989). Although the explorers of the relevant parts of the HR diagram have been many, the results we are interested in, namely $M_{H}$ and $M_{H e}$ on the remnant WD, are so much dependent on detailed post-AGB evolution that we can identify several regions of the HR diagram which we can label by "hic sunt leones", here are the lions, the sign our roman ancestors would put on their geographic maps on the non-explored regions. In fact in these regions it is necessary to approach a number of physical processes not clear from first principles, or numerically difficult to be handled, or which require hydrodinamic computations still completely lacking. "Lions" in a short list are:

i) mass loss phenomena and planetary nebula ejection on the AGB (Schönberner 1979, 1983, 1987, Mazzitelli and D'Antona 1986a);

ii) study of late He-shell pulses and possible consequences of mixing between $\mathrm{H}$-rich and He layers (Fujimoto 1977, Schönberner 1979, Iben et al. 1983, Caloi 1990);

iii) diffusion and explosive H-burning during the first WD phases (Iben and Mc Donald 1985, 1986).

We have listed only reference to computations relevant to the quoted subjects; many others (e.g. including one of us, D'Antona 1989), on the basis of available computations, have speculated on more or less plausible scenarios for the pre-WD evolution.

We need speculations as the standard picture is not satisfactory. In short it is based on the following: the outcoming spectrum of the WD (DA type -hydrogen dominatedor non-DA type -helium dominated) depends on the phase, $\left(\Phi=M_{\text {buff }} / \Delta M_{h}\right)$ during the pulse, at which the evolution off the AGB towards the blue begins, due to planetary 
nebula ejection or to the reaching of a critical minimum mass of the envelope. Iben (1989) summarizes:

i) $0.15 \lesssim \Phi \lesssim 0.75$ : the resulting PNN evolve into DA WDs, with a thick, or evolutionary H-envelope $\left(\mathrm{M}_{\mathrm{H}}=\mathrm{M}_{\mathrm{ev}} \sim 10^{-4}\right.$ for a $0.6 \mathrm{M}_{\odot}$ core $)$

ii) $0.75 \lesssim \Phi \lesssim 1$ : a late TP will occur, either in the PNN phase, or when the star is already close to the WD stage; in this latter case mixing of the $\mathrm{H}$ - and He-envelopes occurs, with not well predicted consequences. In any case, a resulting emergent spectrum devoid of hydrogen is highly probable for the WD;

iii) $0 \lesssim \Phi \lesssim 0.15: \mathrm{M}_{\text {buff }}$ is so thin that a self-induced nova is expected to occur during the first phases of WD evolution, due to the explosive burning of the tail of the hydrogen envelope diffusiong into the helium layer with the tail of the carbon diffusing outward of the core (Iben and Mc Donald 1986). According to Iben, $\mathbf{M}_{\mathbf{H}} \cdot$ must be large enough for this event, but our feeling is that there are not enough computations to be sure of what is the real influence of this phenomenon on the final WD spectral type. In any case, this process will leave $M_{H} \ll M_{\text {ev }}$.

The consequences are:

1) the bulk of DAs have evolutionary $M_{H}$. Wind mass loss in the hot PNN phases is not expected to be able to get rid of most of $\mathrm{M}_{\mathrm{H}}$.

2) some (a few) DAs may have $M_{H}$ lower by orders of magnitude due to mass loss following late TPs of self-induced nova.

3) non-DAs ( $\sim 20 \%$ of all WDs) are formed by a fraction of the stars going through channel (ii).

We know that a series of independent problems connected to WD evolution seem to demand that the bulk of DAs have $\mathrm{M}_{\mathbf{H}} \ll \mathrm{M}_{\mathrm{ev}}$. This is summarized for instance by Fontaine and Wesemael (1990) in this same conference. As Iben (1989) remarks, if we want to have $\mathrm{M}_{\mathrm{H}} \ll \mathrm{M}_{\mathrm{ev}}$ for most DAs, we have to ask that most of $A G B$ stars lose their envelope and begin the post-AGB evolution at $\Phi \sim 0$, that is either at the start of the helium TP or during the phase of quiescent helium burning. In fact, only if the hydrogen shell is not active loss of practically the entire hydrogen envelope can be achieved.

\section{A new suggestion for a solution}

Inside the standard scheme for pre-WD evolution is hidden a main hypothesis: the loss of the envelope which ends the AGB evolution is not correlated with $\Phi$. Can we reject this assumption? For instance, is it more probable to begin post-AGB evolution at the peak of the flash, as the mass loss rate is much larger? On the contrary, the duration of the peak phase is always so short with respect to the interflash period that the mass lost at the peak is always very small. This is exemplified in figure 1 for our typical pulse, but it is true also for small core masses (D'Antona and Mazzitelli 1989, Caloi 1989). Furthermore, the cumulative amount of mass lost during the stationary helium burning is smaller than that lost during the other phases (bottom of figure 1), as on average the luminosity is lower. The computed models shown in figure 1 refer to a typical mass loss rate of Reimer's type. Even if a stronger dependence on the luminosity is assumed, as suggested by recent observations (Oloffson et al. 1990 suggest $d \log \dot{M} / d \log L \sim 4$ ) the results do not change drastically, as the luminosity at peak of the pulse $\left(L_{\text {peak }}\right)$ is larger only by a factor 2 than the average stellar luminosity ( $\left.\mathrm{L}_{\text {average }}\right)$.

Another possibility is the following: probably there is a treshold luminosity $\left(L_{P N}\right)$ above which the wind changes qualitatively, increasing its rate by orders of magnitude. The AGB stars overcome $L_{P N}$ for the first time at $L_{\text {peak }}$, but also this is not enough to trigger the post-AGB evolution, unless the mass loss rate at $L>L_{P N}$ is quite extreme (Schönberner 1987). 
Recent computations of TPs adopting a better numerical approach (Mazzitelli 1990) lead us to suspect a possible way out from the standard scheme. We find that when a very small hydrogen layer is left on the AGB star, prior to the evolution to the blue, during the $T P$ we get $\mathrm{L}_{\text {peak }} \gg \mathrm{L}_{\text {average }}$. In fact the delay between the start of helium pulse and its appearence at the surface (points 1 and 4 in figure 1) is due to the buffer of matter in between the helium shell and the surface. Expansion of this matter absorbs most of the energy liberated by helium burning, and only a small increase of total luminosity occurs. But, when the envelope mass is very small -when the thermal timescale of the envelope is therefore small- as soon as the pulse starts also the surface will be affected. This provides a strong warning against stellar models in which the gravitational energy generation $\left(\epsilon_{g}\right)$ of the outer layers (up to the optical photosphere) is not taken into account (all published results up today). We are making trial computations of TPs for low envelope masses both considering $\epsilon_{g}$ up to the surface or neglecting it for the outer few $10^{-4} \mathrm{M}_{\odot}$, and the results are substantially different! Of course, the numerical problems to be overcome will require still much work, but we can confidently assume that these results will change our view of the latest phases of the stellar evolution.

Our suggestion is the following: for stars of low initial masses, for which during the AGB the envelope results reduced to a few per thousands of solar masses, the last pulse will produce such a large increase of stellar luminosity that these stars leave the AGB at the peak of the pulse, either because $L_{\text {peak }}$ overcomes the $L_{P N}$, or because, in any case, $\dot{M}$ is so much larger that, even in the short time interval of the peak, it is much more probable to lose the entire remaining envelope. During the further wind mass loss, at high $T_{\text {eff }}$, these stars can be able to lose practically the entire hydrogen envelope, as the hydrogen shell is not ignited, and will evolve either into non-DA WDs or into DAs with thin or extremely thin $\mathbf{M}_{\mathbf{H}}$.

The outlined scheme can work when the envelope mass had been reduced to small values by wind, before other mechanisms for the loss of the envelope (e.g. due to the interaction between pulsations and mass loss (Willson 1987)) appear. The progenitor mass must then be small. Surely globular cluster stars or stars like the sun in the disk of our Galaxy would be in this condition. Tentatively, we suggest that all stars which pass through the helium core flash $\left(\mathrm{M} \lesssim 2 \mathrm{M}_{\odot}\right)$ reach this stage. Planetary Nebulae emerging from this kind of evolution would be the result of the interaction of the fast wind of the hot PNN with the slow wind ejected during the AGB phase (Kwok 1983).

At $\mathrm{M}_{\text {prog }} \gtrsim 2 \mathrm{M}_{\odot}$, stars do not experience strong winds in the hydrogen shell burning phase (helium burning in the non degenerate core is ignited at relatively low luminosity). At the start of the AGB phase their envelope is then much more massive than the envelope of $\mathrm{M}_{\text {prog }} \lesssim 2 \mathrm{M}_{\odot}$ which suffer the He-core flash; they have more time to increase the CO core mass and raise in luminosity, until global mechanisms for the envelope ejection come into play. In this perspective, at $\mathrm{M}_{\text {prog }} \gtrsim 2 M_{\odot}$ the standard scheme of pre-WD evolution described in section 4 should in any case apply. Of course, the late-TP and the self-induced nova scenarios must be reconsidered in detail for stars of core masses somewhat larger than $0.6 \mathrm{M}_{\odot}$, descendants of $\mathrm{M}_{\text {prog }} \gtrsim 2 \mathrm{M}_{\odot}$.

An observational consequence of the preceding suggestion appears immediately: the least massive PNN are possibly helium rich, and hydrogen rich PNN average mass should be larger than the average WD mass, as these nuclei are the result of PN ejection from $\mathrm{M}_{\text {prog }}>2 \mathrm{M}_{\odot}$. This latter prediction seems to be observationally confirmed (Mendez et al. 1988). Also Schönberner's (1986) suggestion that H-rich PNN behave like H-burners would not be in contradiction with this scheme: we are stating that H-burning PNN exist, but come out only from $\mathrm{M}_{\text {prog }} \gtrsim 2 \mathrm{M}_{\odot}$, and do not constitute most of WD progenitors. 


\section{Comments on massive progenitors}

The general picture of AGB evolution implies that, during the TP phase, while the CO core increases in mass and the stellar luminosity increases, the third dredge up is able to transform the AGB giants into Carbon stars. It became clear in the course of latest years that there are no carbon stars at $M_{b o l} \lesssim-6$ (Reid and Mould 1985, Mould and Aaronson 1986), but there are more luminous $M$ supergiants, s-type stars, as discovered in the Magellanic Clouds by Wood et al 1983. Recently, these same supergiants have been found to be Lithium rich (Smith and Lambert 1989, 1990). The emerging picture of evolution implies that the AGB phase for thermally pulsing stars which become Carbon rich by the action of the third dredge up, is terminated at $\mathbf{M}_{\mathrm{bol}} \sim-6$, that is for CO core masses of $\sim 0.85 \mathrm{M}_{\odot}$.

Theoretical reasons why the AGB terminates at $\mathrm{M}_{\text {core }} \sim 0.85 \mathrm{M}_{\odot}$ have been described by Wood and Faulkner (1986) and Mazzitelli (1987, 1989), and are correlated to the very low densities obtained at the base of the H-envelope during the peak luminosity phase of TP when the core mass becomes so large: we expect again for these progenitors a sudden loss of the envelope at the peak of the pulse. Thus, also for stars for which the Planetary Nebula phenomenon has not occurred before for different reasons -not linked, consequently, to the TP phase- we expect ejection of the envelope at $L_{\text {peak }}$ and consequent evolution towards non-DA WDs or DAs with very thin $\mathbf{M}_{\mathbf{H}}$.

The most luminous $M$ supergiant are then identified with progenitors massive enough to begin their AGB phase at $\mathrm{M}_{\mathrm{bol}} \lesssim-6\left(\mathrm{M}_{\mathrm{prog}} \gtrsim 5 \mathrm{M}_{\odot}\right)$. They probably do not survive the AGB phase so long that they can become Carbon stars: in fact, as soon as they have experienced the second dredge up, their structure is similar to the previously described one: very cold and expanded envelopes, likely to be lost either before the TP phase or just after a few pulses. This may be an iterative process: in fact, either the hydrogen remnant after the envelope detachement is already very small, or it will reignite. At the following TP the same (or worse) conditions will be present, causing ejection of further hydrogen, until the possible H-remnant becomes in any case extremely small.

\section{Conclusions}

The resulting picture of pre-WD evolution is the following:

1) $\mathrm{M}_{\text {prog }} \lesssim 2 \mathrm{M}_{\odot}$ : we propose envelope ejection at $\mathrm{L}_{\text {peak }}$, which becomes $\gg \mathrm{L}_{\text {average }}$ if wind mass loss has been the only responsible for reduction of the AGB envelope to very low $\left(\lesssim 0.003 \mathrm{M}_{\odot}\right)$ masses;

2) $2 \lesssim M_{\text {prog }} \lesssim 5 M_{\odot}$ : we propose ejection at any TP phase: the standard scheme (e.g. Iben 1989) must work here, but further investigation is required, at least for the role of late TPs and of the self-induced nova mechanism;

3) $\mathrm{M}_{\text {prog }} \gtrsim 5 \mathrm{M}_{\odot}$ : envelope loss at $\mathrm{L}_{\text {peak }}$ for the AGB stars which reach $\mathrm{M}_{\text {core }} \gtrsim 0.85 \mathrm{M}_{\odot}$, or at the second dredge up, or after a few pulses for the most massive $\mathbf{M}_{\text {prog }}$ has already been proposed (Mazzitelli 1987). These stars also may evolve into WD having very thin H-envelopes.

\section{References}

Barnes, C. A. 1988. In Advances in Nuclear Astrophysics" eds. E. Vangioni-Flam, J. Audouze, M. Casse, J.P. Chieze and J. Tran ThanhVan, p. 205

Bergeron, P, Saffer, R., Liebert, J. 1990. In "Confrontation between stallar pulsation and evolution, ed. C. Cacciari (Provo UT: Astronomical Society of Pacific) in press 
Caloi, V. 1989. Astron. Astrophys. 221: 27

Caloi, V. 1990. Astron. Astrophys. 232: 67

Caughlan, G. R., Fowler, W. A. 1988. Atomic Data and Nuc. Data Tables 40: 283

Cox, J. P. 1980. "Theory of Stellar Pulsation", Princeton University Press: Princeton

D'Antona, F. 1987. Mem.S.A.It. 58: 123

D'Antona, F. 1989. "White Dwarfs" IAU Coll. 114 ed. G. Wegner Springer: Berlin, p. 44

D'Antona, F., Mazzitelli, I. 1989. Ap. J. 347: 934

D'Antona, F., Mazzitelli, 1990. Ann. Rev. Astron. Astrophys. 28

Fowler, W., Caughlan, G. R., Zimmerman, B. A. 1975. Ann. Rev. Astron. Astrophys. 13: 69

Fontaine, G., Wesemael, F. 1987. "The Second Conference on Faint Blue Stars" IAU Coll. 95 , ed. A. G. Davis Philip, D. S. Hayes and J. W. Liebert, L. Davis Press Inc. Schenectady, New York, p. 319

Fontaine, G., Wesemael, F. 1990. In this conference

Fujimoto, M. Y. 1977. Publ Astron. Soc. Jpn. 29: 331

Garcia-Berro, E., Hernanz, M., Isern, J., Mochkovitch, R. 1988. Astron. Astrophys. 193: 141

Harris, M. J., Fowler, W. A., Caughlan, G. R., Zimmerman, B. A. 1983. Ann. Rev. Astron. Astrophys. 21: 165

Iben, I. Jr. 1984. Ap. J. 277: 333

Iben, I. Jr. 1989. in Evolution of Peculiar Red Giant Stars, IAU Coll. 106, eds. H.R. Johnson \& B. Zuckerman, Cambridge University Press, p. 205

Iben, I. Jr., McDonald, J. 1985. Ap. J. 296: 540

Iben, I. Jr., McDonald, J. 1986. Ap. J. 301: 164

Iben, I. Jr., Kaler, J. B., Truran, J. W., Renzini, A. 1983. Ap. J. 264: 605

Kepler, S. O., Vauclair, G., Dolez, N., Chevreton, M., Nather, R. E., Winget, D. E., Provencal, J. L., Clemens, J. C., Fontaine, G. 1990. Ap. J. 357: 204

Kwok, S. 1987. Proceedings of the Calgary Workshop "Late stages of stellar evolution" Eds. S. Kwok and S. R. Pottasch Reidel, Dordrecht, p. 321

Mazzitelli, I. 1987. Mem.S.A.It. 58: 117

Mazzitelli, I. 1989. "White Dwarfs" IAU Coll. 114 ed. G. Wegner Springer: Berlin, p. 29

Mazzitelli, I. 1990. in preparation

Mazzitelli, I., D'Antona, F. 1986a. Ap. J. 308: 706

Mazzitelli, I., D'Antona, F. 1986b. Ap. J. 311: 762

Mazzitelli, I., D'Antona, F. 1987. "The Second Conference on Faint Blue Stars" IAU Coll. 95 , ed. A. G. Davis Philip, D. S. Hayes and J. W. Liebert, L. Davis Press Inc. Schenectady, New York, p. 351

Mazzitelli, I., D'Antona, F. 1990. In preparation

Mendez, R. H., Kudritzki, R. P., Herrero, A., Husfeld, D., Groth, H. G. 1988. Astron. Astrophys. 190: 113

Mould, J. R., Aaronson, M. 1986. Ap. J. 303: 10

Nather, R. E., Winget, D. E., Clemens, J. C., Hansen, C. J., Hine, B. P. 1990. Ap. J.361: 309

Nomoto, K. 1984. Ap. J. 277: 791

Oloffson, H., Carlström, U., Eriksson, K., Gustaffson, B., Willson, L. A. 1990. Ap. J. 361: 309

Pelletier, C., Fontaine, G., Wesemael, F., Michaud, G., Wegner,G. 1986. Ap. J. 307: 242

Reid, N., Mould, J. 1985. Ap. J. 299, 236

Schönberner, D. 1979. Astron. Astrophys. 79: 108

Schönberner, D. 1981. Astron. Astrophys. 103: 119

Schönberner, D. 1983. Ap.J. 272: 708

Schönberner, D. 1986. Astron. Astrophys. 169: 189 
Schönberner, D. 1987. Proceedings of the Calgary Workshop "Late stages of stellar evolution" Eds. S. Kwok and S. R. Pottasch Reidel, Dordrecht, p. 337

Smith, V. V., Lambert, D.L. 1989. Ap. J. 345: L75

Smith, V. V., Lambert, D.L. 1990. preprint

Stevenson, D. J. 1980. J. Phys. Suppl. 41: C2-61

Truran, J. W., Livio, M. 1989. IAU Coll. 114, "White Dwarfs" ed. G. Wegner, Springer: Berlin, p. 498

Weidemann, V., Koester, D. 1984. Astron. Astrophys. 132 :195

Werner, K., Heber, V., Hunger, K. 1990. Aston. Astrophys., in press

Willson, L. A. 1987 Proceedings of the Calgary Workshop "Late stages of stellar evolution" Eds. S. Kwok and S. R. Pottasch Reidel, Dordrecht, p. 253

Williams, R. E. 1985. "Production and distribution of CNO elements" ed. I. J. Danziger, Garching, ESO, p.225

Winget D., Van Horn, H. M. 1987 "The Second Conference on Faint Blue Stars" IAU Coll. 95 , ed. A. G. Davis Philip, D. S. Hayes and J. W. Liebert, L. Davis Press Inc. Schenectady, New York, p. 363.

Wood, P. R., Bessell, M. S., Fox, M. W. 1983. Ap. J. 272: 99

Wood, P. R., Faulkner, D. J. 1986. Ap. J . 307: 659 